\title{
Les PEARLS (Practical Evidence About Real Life Situations) du groupe Cochrane Pre-hospital and Emergency Care
}

\author{
P. Jabre \\ (C) SFMU et Lavoisier SAS 2018
}

\section{Les tests cliniques prédictifs de difficultés de gestion des voies aériennes ont une efficacité limitée}

Roth D, Pace NL, Lee A, et al (2018) Airway physical examination tests for detection of difficult airway management in apparently normal adult patients. Cochrane Database Syst Rev 5:CD008874

\section{Problématique}

La gestion des voies aériennes (GVA) est primordiale dans la prise en charge des patients inconscients, anesthésiés ou qui nécessitent une sédation. Une ventilation insatisfaisante ou une intubation difficile sont des situations délétères et difficiles à prévoir y compris chez les patients qui ne présentent pas de pathologies ou d'anomalies anatomiques des voies aériennes supérieures.

La difficulté dans la GVA est définie par la présence d'un de ces critères : difficulté de ventilation au masque, difficulté de laryngoscopie directe, difficulté d'insertion de la sonde d'intubation ou échec de l'intubation trachéale.

Plusieurs tests cliniques existent pour dépister les situations à risques de GVA.

\section{Objectifs}

Quel est le test clinique de dépistage le plus adapté pour prédire une difficulté dans la GVA chez l'adulte qui ne présente aucune anomalie anatomique des voies aériennes?

P. Jabre $(\triangle)$

Cochrane Pre-hospital and Emergency Care,

103, boulevard de Magenta, F-75010 Paris, France

e-mail : patricia.jabre@aphp.fr

UMR Inserm U970 (PARCC), université Paris-Descartes, 58, rue Leblanc, F-75015 Paris, France

Samu de Paris, hôpital Necker-Enfants-malades, AP-HP, 149, rue de Sèvres, F-75730 Paris cedex 15, France

\section{Résultats principaux}

En ce qui concerne les difficultés de laryngoscopie directe : le test de morsure de la lèvre supérieure à la sensibilité la plus élevée comparativement aux autres tests (67\%). Les scores de Wilson et le test modifié de Mallampati ont les spécificités les plus élevées (95\% et $80 \%$ ).

En ce qui concerne les difficultés d'intubation : le test de Mallampati modifié présente la plus haute sensibilité de tous les tests étudiés, significativement plus élevée que le test de l'ouverture de la bouche et de la distance thyromentonnière $(\mathrm{P}<0.001)$. Malheureusement, les informations ne sont pas suffisantes pour le comparer au test de morsure de la lèvre supérieure. Le test d'ouverture de la bouche montre de façon significative la plus haute spécificité $(\mathrm{P}<0.001)$.

En ce qui concerne la difficulté de ventilation au masque et l'échec d'intubation : les données ne sont pas suffisantes pour effectuer des comparaisons de qualité.

\section{Commentaires}

Tous les tests de dépistage examinés présentent une sensibilité basse et une grande variabilité. Ainsi la fréquence élevée de faux négatifs peut amener à des situations catastrophiques notamment lors de l'induction d'une anesthésie.

La qualité méthodologique des études diagnostiques retenues est jugée élevée en ce qui concerne l'applicabilité et modérée pour le risque de biais. L'analyse et la présentation des études qui portaient sur les combinaisons de test n'ont $\mathrm{pu}$ être réalisées du fait d'une forte hétérogénéité non expliquée.

Les tests exploités dans cette revue mettent en évidence une efficacité relative dans les situations contrôlées ou hospitalières. Il n'y a pas d'analyse concernant le recours à ces tests en situation d'urgence ou en préhospitalier où le ratio de difficulté d'intubation est bien plus élevé qu'en situation hospitalière. 
D. Meyran

daniel.meyran@me.com

Service mobile d'urgence et de réanimation,

Bataillon de Marins Pompiers de Marseille,

groupement santé, F-13233 Marseille, France

J. Dumouchel

dumouchel.julie76@yahoo.fr

Département de médecine d'urgence,

CHU de Tours, F-37000 Tours, France

\section{L'aspirine, par voie orale, pourrait être intéressante dans la prise en charge des accès de céphalée de tension épisodique}

Derry S, Wiffen PJ, Moore RA (2017) Aspirin for acute treatment of episodic tension-type headache in adults. Cochrane Database Syst Rev 1:CD011888

\section{Problématique}

La céphalée de tension est la première cause de céphalées au monde. Bien que bénigne, elle entraîne un coût important pour la société (consultations, arrêts de travail).

\section{Objectifs}

L'aspirine, par voie orale, est-elle efficace et bien tolérée chez l'adulte souffrant d'accès fréquents (2 à 14 épisodes par mois) de céphalée de tension épisodique d'intensité modérée à sévère ?

\section{Résultats principaux}

Le soulagement complet à 2 heures, critère de jugement proposé par l'International Headache Society, n'a pas pu être étudié par manque de données. Le recours à un médicament de secours est significativement moins important avec l'aspirine par rapport au placebo (faible niveau de preuve). Le niveau de satisfaction des patients est supérieur sous aspirine par rapport au placebo (très faible niveau de preuve). En termes d'effets indésirables, l'aspirine est aussi bien tolérée que le placebo (faible niveau de preuve).

\section{Commentaires}

Les résultats de cette méta-analyse se limitent à une population très précise (adultes ayant des céphalées de tension fréquentes, d'intensité modérée à sévère). Les effectifs restreints ne permettent pas de privilégier une dose d'aspirine (500, 650 ou $1000 \mathrm{mg})$. La tolérance de ce traitement n'a été évaluée que pour une prise unique d'aspirine et à très court terme $(<24 \mathrm{~h})$. Cependant, les auteurs précisent que compte tenu du faible prix et de l'accessibilité de ce traitement, l'aspirine pourrait avoir un intérêt, particulièrement dans les pays en voie de développement.

\author{
S. Beroud \\ sebastien.beroud@chu-lyon.fr \\ Service d'accueil des urgences, CHU Lyon-Sud, \\ F-69394 Lyon, France \\ Y. Auffret \\ y.auffret@ch-cornouaille.fr \\ Urgences, centre hospitalier de Cornouaille, \\ F-29107 Quimper, France
}

\section{Oxygénothérapie dans le traitement des crises migraineuses et des algies vasculaires de la face : faible niveau de preuve}

Bennett MH, French C, Schnabel A, et al (2015) Normobaric and hyperbaric oxygen therapy for the treatment and prevention of migraine and cluster headache. Cochrane Database Syst Rev 12:CD005219

\section{Problématique}

Les migraines et les algies vasculaires de la face (AVF) sont des pathologies handicapantes. Sept $\%$ des hommes et $18 \%$ des femmes souffrent de crises migraineuses et $0,2 \%$ de la population souffre de crises d'AVF. Plusieurs traitements sont disponibles à la phase aiguë et en traitement de fond.

\section{Objectif}

Quelle est l'efficacité et la tolérance de l'oxygène hyperbare (OHB) et normobare (ONB) lors de crises de migraine et d'AVF ? L'oxygène (OHB ou ONB) diminue-t-il les récidives?

\section{Résultats principaux}

Concernant la migraine, l'OHB semble efficace pour soulager les crises migraineuses (faible niveau de preuve). Une seule étude montre la supériorité de l'OHB à l'ONB. L'OHB ne semble pas diminuer le nombre de récidives, les nausées ou vomissements, ni le recours à d'autres thérapeutiques.

Concernant les AVF, l'OHB semble peu efficace pour soulager les crises d'AVF (1 seul essai de faible puissance). Par contre, l'ONB semble efficace pour soulager les crises en comparaison au placebo, mais non supérieur à l'ergotamine. Aucune étude n'a évalué l'effet de l'OHB ou l'ONB sur les récidives.

Quant à la tolérance, aucun effet indésirable grave n'a été noté avec l'OHB ou l'ONB. Les effets indésirables 
rencontrés avec l'OHB étaient rares (claustrophobie, barotraumatisme de l'oreille, ...).

\section{Commentaires}

Cette revue systématique d'essais randomisés repose sur de faibles effectifs, et les résultats n'ont pas systématiquement pu être sommés du fait de l'hétérogénéité existante. Le problème de l'OHB est son accessibilité et son coût. Ce traitement pourrait être une alternative pour les patients migraineux réfractaires à tous les autres traitements. Des études sont nécessaires pour savoir si l'OHB est efficace dans ce sous-groupe de patients. De plus, des études seraient intéressantes pour vérifier l'intérêt et les modalités d'administration (débit, durée) de l'ONB dans les AVF.

Y. Auffret

y.auffret@ch-cornouaille.fr

Urgences, centre hospitalier de Cornouaille, F-29107 Quimper, France

S. Beroud

sebastien.beroud@chu-lyon.fr

Service d'accueil des urgences, CHU Lyon-Sud, F-69394 Lyon, France 